\title{
Adaptation Capability of Cognitive Map Improves Behaviors of Social Robots
}

\author{
Abdelhak Chatty ${ }^{1,2}$,Philippe Gaussier ${ }^{2}$, Ilhem Kallel ${ }^{1}$, Philipe Laroque $^{2}$, and Adel M. Alimi ${ }^{1}$ \\ ${ }^{1}$ REGIM: REsearch Groups on Intelligent Machine \\ University of Sfax, National School of Engineers (ENIS) Sfax, Tunisia \\ ${ }^{2}$ ETIS: Neuro-cybernetic team, Image and signal Processing \\ National School of Electronics and its Applications (ENSEA), Cergy-Pontoise University, Paris, France \\ \{abdelhak_chatty, ilhem.kallel, adel.alimi\}@ieee.org, Gaussier@ensea.fr, laroque@u-cergy.fr
}

\begin{abstract}
In this paper, we study the impact of the cognitive map's adaptation in the context of multi-robot system. This map governs the emergence of non-trivial behaviors and structures at both individual and social levels. In particular, we show that adding a simple imitation and deposit behavior allows the cognitive robots to adapt themselves in unknown environment to solve different navigation tasks. We show that in our architecture the individual discoveries in each robot (i.e., goals) can have an effect at the population level, which induce then a new learning at the individual level and reciprocally, from the individual to the population level. We performed a series of experimentations with robots and simulated agents to validate our system.
\end{abstract}

\section{INTRODUCTION}

Emergent behavior is one of the main topics of research in the field of social robotics. For instance, in an unknown environment, interaction between robots can be based on stigmergy [1] which means indirectly communicating through the environment changes. In this kind of environment, the memory is the environment itself, however we believe that the use of a cognitive map in each robot is essential when the robot cannot leave a physical trace. Therefore, our aim is to put on light the adaptive capability of a cognitive map to solve at the same time a navigation task and to solve a deposit problem. The cognitive map can help to lead the emergence of non-trivial structures and behaviors in a Cognitive multi-robot system (CMRS). Many models based on reactive agents/robots were proposed in this area. For example [2] has proposed a model relying on biologically plausible assumptions to account for the phenomenon of dead body deposit in ants. [3] shows that acting on objects simplifies the reasoning needed by multi-robot systems and allows deposit of scattered objects. In [4] the authors studied the deposit system and sorting of colored frisbees by a multi-robot system. In [5] the author studied the deposit of small cylinders by a group of agents. In comparison to these previous works, we propose a solution which is use of cognitive maps and local rules to allow our robots to develop different emergent behaviors. The remainder of this paper is organized as follows: section 2, describes the bio-inspired architecture of our cognitive map. Section 3 depicts the structure, building and the adaptive capability of the cognitive map. Section 4 describes the building of the emergent deposit system. We analyze in seection 5 how the robots self-organize in emergent structures. The last section concludes the paper.

\section{THE BIO-INSPIRED ARCHITECTURE}

Each robot in our cognitive multi-robot system is able to learn and to create its own cognitive map online, whose structure depends on their own experience and discovery of the environment in which they live, based on bio-inspired architecture. Starting from neurobiological hypotheses on the role of hippocampus in the spatial navigation, [6] form a model of the cognitive map in the hippocampus representing the entire environment and not only the shortest paths to a given goal. The model proposed by [7] provides a complete neural architecture of the learning process and use of a cognitive map and the association with a mechanism of action selection. [8] also propose a model of cognitive map in the prefrontal cortex, which is based on cortical columns. in the same ideas biologically inspired, several works [9], [10] brought to light special cells in the rats hippocampus that fire when the animal is at a precise location. These neurons have been called place cells (PC). We do not directly use them to navigate, plan or construct a map, we rather use neurons called transition cells (TC)[11]. This kind of cells are inspired by a neurobiological model of temporal sequences learning in the hippocampus [12], [13], [14]. A transition cell codes for a transition between two PCs successively winning the competition, respectively at time $\mathrm{t}$ and $\delta t$. The model [15], which presents the role of the hippocampus, shows us the architectures grounding using the transitions between locations. The entorhinal cortex (EC) receives signals from associative cortical ranges and then it filters and merges this multimodal information in order to transfer it, on one hand to pyramidal cells (CA3), and on the other to the dentate gyrus (DG). The DG operates an eminence between the signals and puts together a temporal hierarchy which later on is retransmitted on CA3 cells. This temporal hierarchy allows CA3 to be aware of past events and put them in correspondence with present events, therefore behaving like an associative memory through stocking possible transitions between these events. The recognition of the ongoing sequence happens at the level of CA1 using EC and CA3 information. This information is then sent to the Subiculum to be processed, independently from the robots orientation, through the heads 
executive cells. It also departs to the prefrontal cortex (PRC) to serve the higher levels of cognitive processes.

\section{THE COGNITIVE MULTI-ROBOT SYSTEM}

Our interpretation of the bio-inspired model is presented in figure 1: the signals provided by EC are solely spatial and consistent with spatial cells activities. Spatial cells activities are calculated from visual information then submitted to a WinnerTake-All competition in order to only select the cell with the strongest response at a specific location. We will subsequently be speaking about the current location by indicating the spatial cell which has the highest activity at a given location. The temporal function at the level of DG is reduced to the mere memorization of past location. The acquired association at the level of CA3 is then the transition from a location to another aside from all information concerning the time put to carry out this transition. Once the association from the past location and the new one is learned, every new entry will reactivate the corresponding memory in the DG.

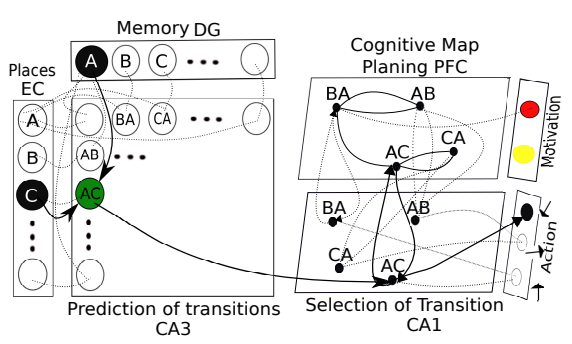

Fig. 1. Model of hippocampo-cortical of the creation of the cognitive map

During exploration of the environment, the cognitive map is gradually created when the robot moves from one place to another. The equations governing learning in the cognitive map are 1:

$\frac{d W_{i j}^{C C}(t)}{d t}=T(t) \cdot\left(\left(\gamma-W_{i j}^{C C}\right) \cdot X_{i}^{C}(t) \cdot X_{j}^{C}(t)-W_{i j}^{C C}(t) \cdot\left(\lambda 1 \cdot X_{j}^{C}(t)-\lambda 2\right)\right)$

$T(t)$ is a binary signal ( 0 or 1$)$ active when a transition is made (moving from one place to another). This signal controls the learning of recurrent connections $W^{C C} \cdot \gamma$ is a parameter less than 1 regulating the distribution of motivation activity in the map. $\lambda 1$ and $\lambda 2$ are parameters of active and passive oblivion respectively on the recurrent connections. $S(t)$ is a signal marking the satisfaction of a goal (for exemple the resources discovery). This signal controls the learning of synaptic connections between neurons in $W^{M C}$ motivations activity $X^{M}$ and neurons of the cognitive map of activity $X^{C}$, where the equation is $d W_{i j}^{M C}(t) / d t=S(t)$, for $i, j=$

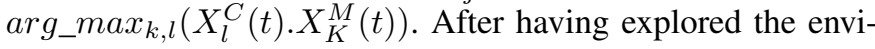
ronment, the robots now are in a position to predict in each situation the locations directly reachable. During a change in winning locations, a neuromodulation signal is transmitted to point out that a transition has just taken place. The achieved transitions activity then resets back towards the PFC. In order to show the robustness of the cognitive map, we try to study initialy, the behavior of a cognitive multi-robot system, based on the same model, capable of learning several goals in unknown environment. Figure 2 presents the initial environment, which is composed by 2 sources (R1 and R2), 2 obstacles(O1 and $\mathrm{O} 2$ ) and 2 identical robots with a threshold of vigilance equal to 0.65 and a duration of the learning equal to 30 minutes.

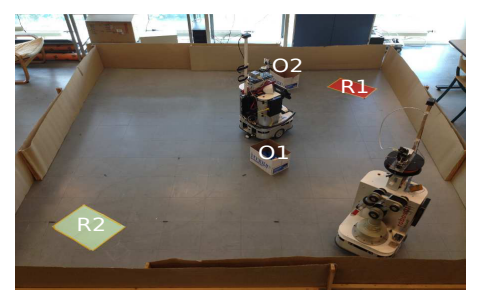

Fig. 2. The cognitive multi-robot system in unknown environment. The video is available in http://perso-etis.ensea.fr/neurocyber/Videos/Cognitive_MultiRobot_System/CMRS

To create the PC, the robot takes, at each moment in time, a visual panorama of the surrounding environment. A monodirectional camera mounted on a pan system allows to make several shots. The views are processed to extract visual landmarks. After learning these landmarks, a visual code is created by combining all the landmarks of a panorama with their azimuth. This configuration serves as a code to PCs. Figure 3 shows that our cognitive robots are able to avoid the stable and dynamic obstacles, to navigate, to learn and to construct on-line and at the same time, their own cognitive map in a unknown environment.

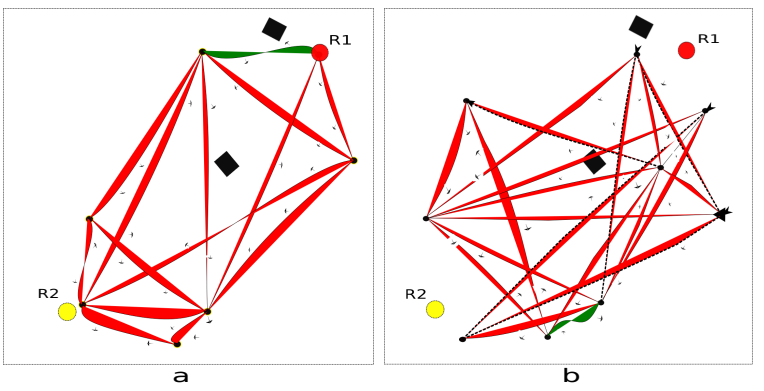

Fig. 3. Simplified view of the cognitive maps of the robots based on transition cells. (a) presents the cognitive map of the first robot and (b) presents the cognitive map of the second robot. The construction process is based on: place recognition using PCs, activation of one PC and delayed activation of another allows for transition prediction (green transition) and the need for sources (R1 and R2) triggers maximum motivation for a given goals.

The shape of the cognitive map in figure 3 , proves that its construction is related to the robot's own perception, that is why the different robots also learn the position of the 2 sources in different manner, and the number of the PCs is not the same ( 7 for the first robot, and 9 for the second with the same threshold of vigilance). The change in the environment (the moving obstacles and the increasing the size of the environment) allows us to show the adaptive capability of the cognitive map. Figure 4a shows the new environment. 
Using previously parameters, figure $4 \mathrm{~b}$ and $\mathrm{c}$ prove that the robots were able to adapt their own cognitive maps to learn the new environment throught adding a new PCs and TCs. This validates the robustness of the cognitive map because the change in the environment has not disturb the robots from learning about the new environment.

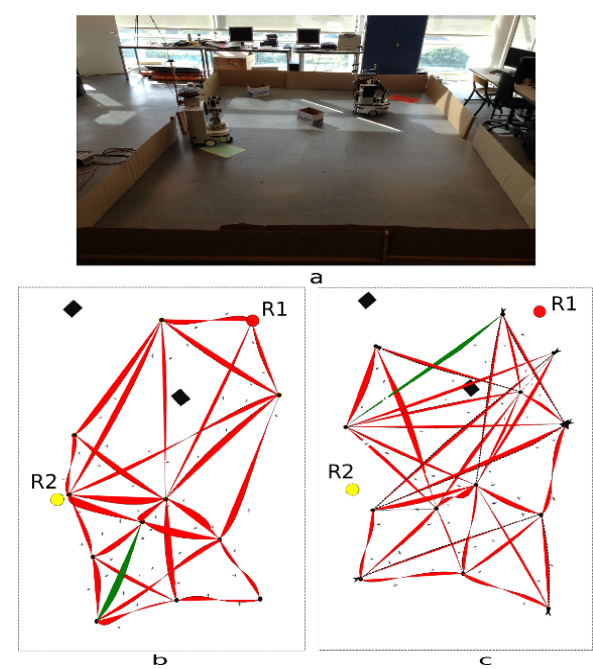

Fig. 4. Cognitive map evolution induced by a changing environment where R1 and R2 are the sources.(a) presents the cognitive map of the first robot and (b) presents the cognitive map of the second robot.

\section{A. The selection of the shortest path}

When discovering a source (food or water), the motivation associated with it (thirst or hunger) is associated with the cognitive map at the location where it was found. This motivation then spreads to the graph, indicating the shortest path to reach the source from whichever location known. We can therefore merge these motivation activities with the transition prediction activities coming from the hippocampus. The level of prediction of different transitions from the hippocampus is substantially the same. The activity derived from the cognitive map comes then to predetermine the transition choice among those that have been predicted, through selecting thereby the transition leading the fastest towards the target. A field of neurons gathers the information on the robots direction during its entire passage of a location. It provides therefore, at the exit of a location, the direction and the length of the trajectory performed at the location. This direction is considered to be the one which is required to perform the transition. During the selection of a particular transition in order to reach the target, we can replicate, in terms of direction, the motion associated which has already been learned during the discovery of the environment. Thus, the robots are able to reset from transition to another by taking the shortest path in order to reach its target. In practice, when the need for a given source arises, the drive triggers the activation of the appropriate goals and throught the cognitive map the appropriate TCs whose destination is the goal location. Figure 5 shows the difusion of the motivation activity of the two robots along the cognitive map combining transition prediction and motivation gives the recognized transition, and the action corresponding to this transition is selected until the TC whose origin codes the robots' actual location.

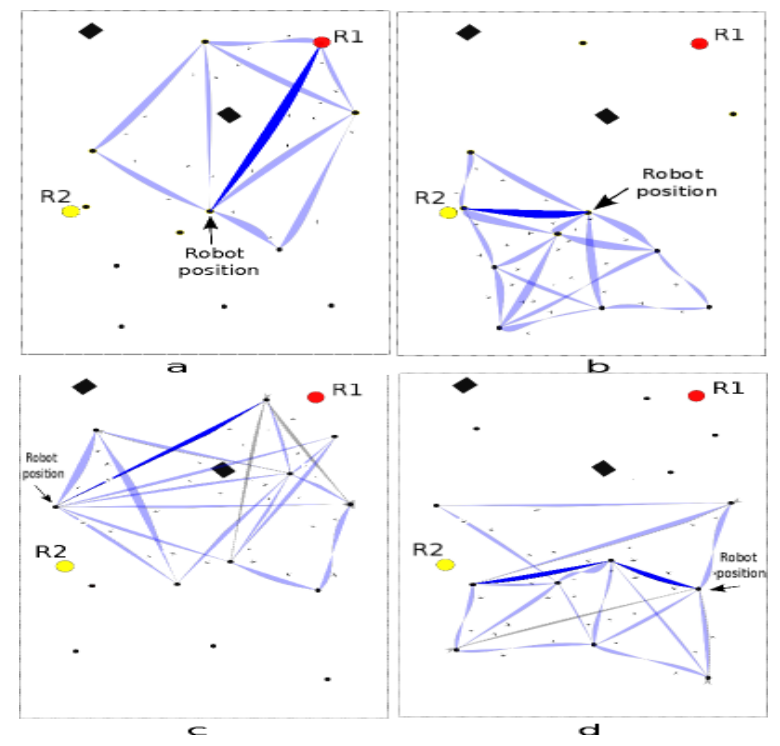

Fig. 5. Diffusion of the motivation along the cognitive map. The robots were able to select the shorest path in order to reach their targets. A and b show how the first robot satisfy its motivations related respectively to the sources $\mathrm{R} 1$ and $\mathrm{R} 2$. $\mathrm{C}$ and $\mathrm{d}$ also show how the second robot satisfy its motivations related respectively to the same sources $\mathrm{R} 1$ and $\mathrm{R} 2$.

The figure also shows the nearest path (dark blue) selected by the robots. Thus, we prove that the cognitive map aims to teach new positions of sources to the robots and evolve in a unknown environment. It allows robots to avoid planning problems before complete exploration of the environment. Moreover, it gives the same results as an ant algorithm[16] without the need to leave physical trace in the environment.

\section{B. Imitation and cognitive map}

We believe that several benefits can be expected from imitation capability, among which the possibility to share partial knowledge of the environment, source locations and so on. In particular, [17] showed that adding an imitation capability can dramatically enhance the survival rate of a multi-agent system. To study the influence of the imitation, we add in our cognitive multi-robot system, a reactive robot which uses an imitation strategy based on the azimuth: if a single robot is visible, it becomes the chosen imitation target; if several robots are visible, then the chosen target is the one that is closest to the direction of the robot who trying to imitate. It is important to mention that the imitated robot is never aware of being imitated. Figure 6a shows the reactive robot (RR) tries to follow one of the cognitive robots (CR1,CR2) which are in navigation task. In the same figure, we see that the reactive robot is able to see the two robots and decides to follow the robot which is the nearest to its own direction. Figure $6 \mathrm{~b}$ show that the reactive robot succeeded to found the first source with following CR2 and after CR1 (the trajectory of robots given 
by Figure 6 allows to explain more the individual and the population behavior of robots). Finally, figures $6 c$, d,e and $\mathrm{f}$ also prove that the imitation strategy allows the reactive robot to find the second source with the help of only CR2. In emergent collective systems, the behavior of an agent is important for the emergence of other behaviors that will help the whole system to solve a specific task. With the use of an imitation strategy we show that it is the system, which is based on the building of a cognitive map, that can help an agent to satisfy its motivations. Thus, adding a simple imitation strategy, to our cognitive architecture, allows the system of multi-robot to help the individual robot.

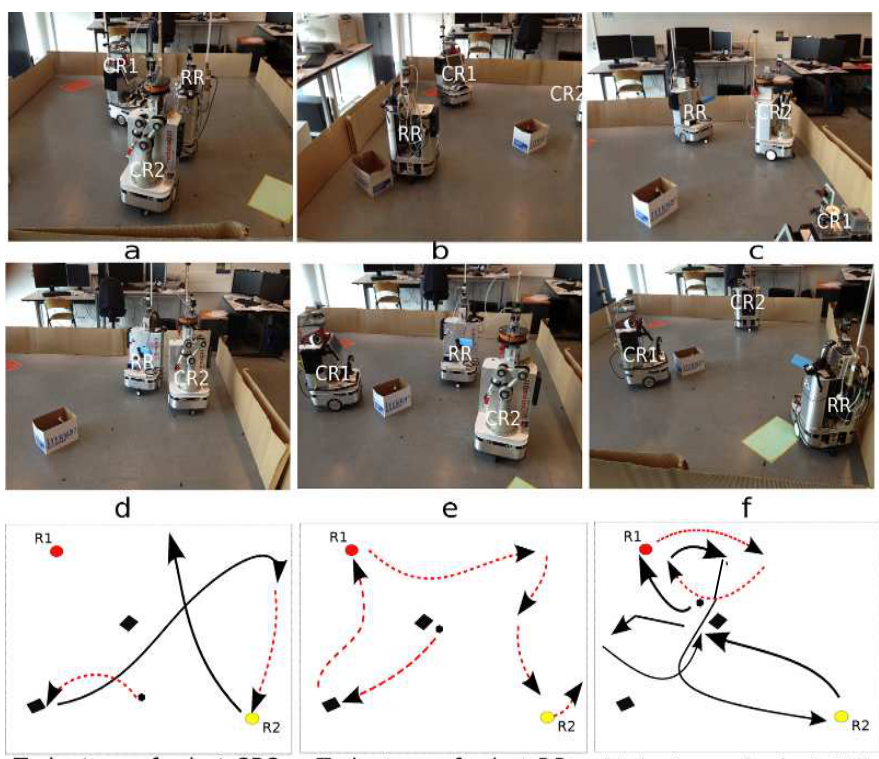

Trajectory of robot CR2 Trajectory of robot RR Trajectory of robot CR1

Fig. 6. The influence of the imitation behavior in multi-robot system. The experiment is done with three robots in the same environment as in figure 2. RR: reactif robot, $\mathrm{CR} 1$ and $\mathrm{CR} 2$ the cognitive robots, $\mathrm{R} 1$ and $\mathrm{R} 2$ are the two resources. The figure is presented with chronological order. The trajectory of the robot shows that RR is able to find the two resources by imitating the trajectory of CR1 and CR2 (the dashed arrows in the trajectory of CR1 and CR2 present the imitated trajectory). The video is available in http://perso-etis.ensea.fr/neurocyber/Videos/Cognitive_MultiRobot_System/Imitation_CRs-RR

\section{The BULDING OF EMERGENT DEPOSIT SySTEM}

To show the adaptive capability of our cognitive map, we add a simple behavior of deposit to our agents. In this part we will use a simulated cognitive multi-agent system (CMAS). The CMAS use the same architecture, rules of learning and the same cognitive properties as the cognitive multi-robot system. The CMAS are motivated by the simulation of three types of needs (hunger, thirst and stress). Each need can be satisfied by a corresponding source, namely food, water and nest, that can be found in the environment. The level of each type of need is internally represented by an essential variable, ei(t) whose value is in $[0 ; 1]$ and varies with time as $d e_{i} / d t=-\alpha_{n} e_{i}(t)$ where, $\alpha_{n}$ represents the decreasing rate of the essential variable. When a satisfaction level $e_{i}(t)$ falls bellow a given $\mathrm{ST}$, the corresponding motivation is trigged so that the agent has to reach a sources allowing to satisfy his needs using their cognitive map. Thus, changing its value has an impact on the frequency of the visits to the sources: the higher $\alpha_{n}$, the more frequent the visits. If no sources of the corresponding type have been found by the agent, the level decreases to 0 and the agent dies. To maintain the satisfaction level of our CMAS, instead of navigation between the threeoriginal sources it is interesting if the accumulation of individual decisions gives birth of the creation of relevant resources. The localisation of resources is important because when agents achieve them easily, they can increase their average satisfaction level and optimize the planning time looking for satisfaction of their motivations. In situated cognition context we believe that stigmergy based on local rules can lead to create emergent structures allowing creation of relevant resources. For this reason, we describe generic local rules where there is a relationship between the number of taken and deposits of resources according to the number of agents perceived. The agent can indeed, tend to favour the location that contain other agents rather than empty regions to create new resources. Thus, the perception of local agents will control the rules of taken and deposits. The condition of taking is computed by $\operatorname{Pr}_{(\text {Taken })}=\exp ^{-\lambda N_{A}}$ : the agent wants different types of resources in the environment. Indeed, the probability that an agent wants to take some resources goods increase when he perceives that the source is less used by other agents. So, the more agents are near a source, the more the probability of taking decreases and vice versa. Where $N_{A}$ is the number of agents in the neighborhood, $\lambda$ is a constant. Equation of deposits $\operatorname{Pr}_{(\text {Deposits })}=\left(1-\exp ^{-\alpha N_{A}}\right) *\left(1-\exp ^{-\beta t}\right)$ describes the condition for deposits as follows: the agent wants more to deposit more when the place is frequented by other agents and when he is far from the original source conducted the transaction of taking, this favors the relevance of the location of resources deposited. The deposit operation is built on the concept of refueling : the agent puts in the resources that already exist. Where $\alpha, \beta$ are environmental factors, $N_{A}$ is the number of agents in the neighborhood and $t$ is the time since the take

\section{Analysis of Emergent Structures}

We use the environment as shown in figure $7 \mathrm{a}$ at $\mathrm{t}=0$ where the original sources are scattered. The agents start to move randomly in the environment, with a limited range visual of perception that can restrict the ability of agents to perceive the environment (see figure7b). When passing on sources (Water, Nest and Food) an agent increases his level of satisfaction and executes the local rule of taking and transport a quantity from the associated product. The probability of taking increases when the agent does not detect other agents next to the sources. If the decision to transport some products is taken, the agent continues his travel. The probability of deposits increases when the agent detects other agents and he moves away from the original sources. This means that the locations chosen for deposit are often common to several agents. Once deposited, goods constitute a new resources (Depot water, Depot nest 
and Depot food) allowing others to increase their satisfaction level. Agents also have the possibility of refueling resources by adding products to them. This allows stability resources in relevant locations close to several agents to fight against loss, since during the refuelling the available products in the resources will increase.

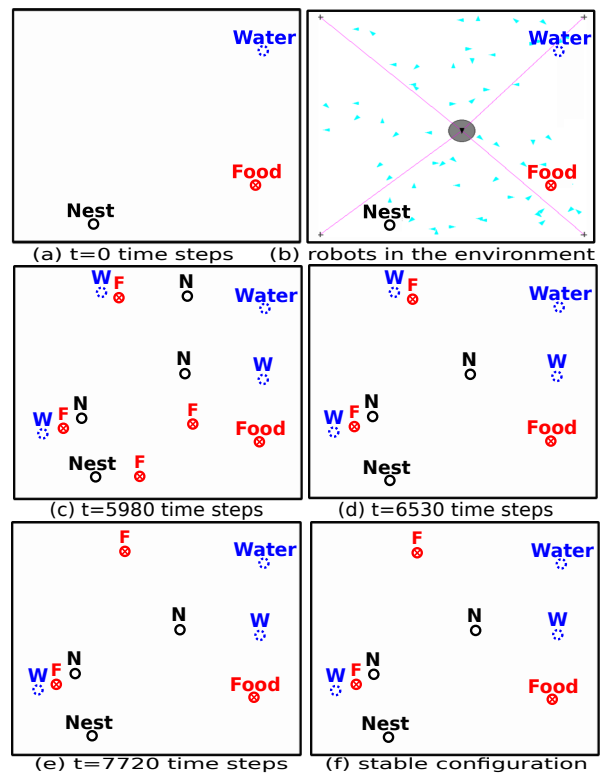

Fig. 7. The figure shows 3 sources Water,Nest and Food providing resources of 3 different kinds (W=Depot water, N=Depot nest and $\mathrm{F}=$ Depot food). The position of the original sources is fixed and they can't disappear from the environment. Through the individual deposit process of agents, resources emerge and become stable after a while. the video is available in http://persoetis.ensea.fr/neurocyber/Videos/Cognitive_Multi-Robot_System/CMAS

However, resources which are abandoned or poorly visited will eventually disappear since the amount of goods available will decrease rapidly. Figures $7 \mathrm{c}, \mathrm{d}$ and e show the disappearance of isolated resources. When a planning agent tries to reach a previously known ressource and realizes that this ressource has disappeared, two things happen (see figure 8):

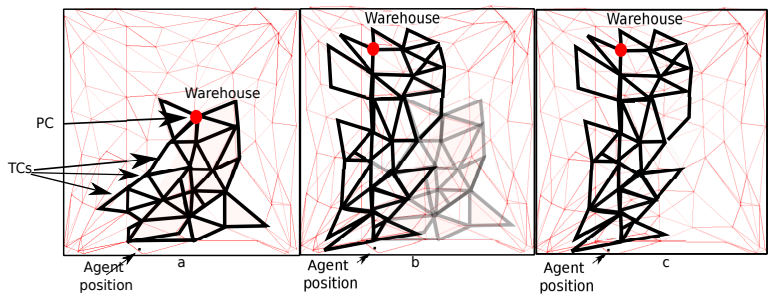

Fig. 8. The evolution of the cognitive map. The PC that encodes the warehouse through time is presented by a red circle and all the paths (TCs) frequented by the agent that lead to the warehouse are reinforced in the cognitive map. (a) The current PC and the TCs that encode and lead respectively to warehouse, (b) The disappearance of the warehouse leads to the dissociation of the PC and the motivation from the old warehouse place. After discovering a new warehouse, the agent acquires (learns) the new place (creation of a new PC) and the TCs that lead to this new place become reinforced. (c) The forgotten of the old place : After a while, the TCs, that lead to the old warehouse, disappear and the new ones become completely reniforced. (i) the agent dissociates the current PC from the formerlycorresponding resource, and (ii) it resets the motivation to 0 . Since the PC does not fire any more when the agent feels the need for this resource, there are chances that the use of transitions leading to this place be progressively forgotten. Similarly, when a new, matching resource is discovered, the paths leading to the resource are rapidly reinforced, making the cognitive map evolve synchronously with the environment.

Figure $7 \mathrm{e}$ shows that the CMAS converges to a stable solution with a fixed number of resources in fixed places for more than 20000 time steps (see figure 7f). Here we note the emergence of stable large resources which in some cases emerge to create resources "villages" (narrow regions containing all 3 resources). Figure 9 shows that until 4500 steps of time, the number of resources is not stable.

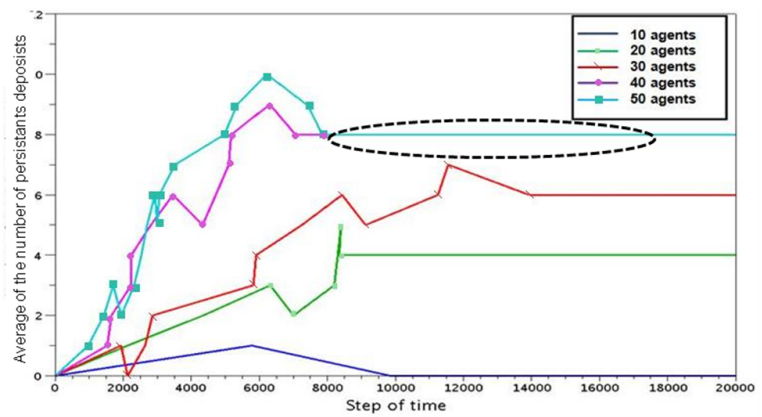

Fig. 9. The average of persistent resources and convergence time in our CMAS. We can see that the average number of resources and the average convergence time stabilized with 40 agents.

Obviously the average of the convergence time increases with the increase of the number of agents. The number of persistent resources varies between 0 and 8 . However, from 40 agents, the average number of resources stabilized at 8 and the average of convergence time stabilized at 7500 time steps. For 10 agents, the emergent number of persistent resources is 0 , this shows that the cooperation between agents is not successful in order to determine the relevant locations in the environment. The analysis of the agent's behavior was able to prove that a fixed number of resources can emerge in fixed positions while minimizing the number's resources and the convergence time. The adaptive cognitive map, allows the CMAS to create emerging resources in relevant places frequented by agents. Note that the agents were able to adapt their environment and created villages of resources, which consist of three types of resources (water Depot, nest Depot and food Depot). The average number of visits to resources (115) is more important than to the original sources (27), which shows that their location is more convaincing. The same behavior also allows to keep a fixed number of resources (here 6) and remove others located in irrelevant places without having to use thresholds to limit the number of resources nor to specify their locations. The adaptive capability of the cognitive map, allows cognitive agents to create an emergent structure which is the stable state for the whole multi-agent system allowing the optimization of planing. Based on the selection of the 
shortest path, table I shows that agents can optimize their planning time with the use of relevant resources. This leads to a higher average satisfaction level. We calculate the average satisfaction level (ASL) according to equation 2. These values show the performance of our CMAS to keep the agents in their comfort zone.

$$
A S L=\frac{\left(\frac{1}{3 i}\right) * \sum_{i=1}^{i=n}\left(S L_{\text {water }_{i}}+S L_{\text {food }_{i}}+S L_{\text {nest }_{i}}\right)}{N}
$$

Where SL is the satisfaction level of sources, $n$ is the number of iterations, $\mathrm{N}$ is the number of expiriments.

TABLE I

OPTIMIZATION OF PLANNING TIME UNTIL 20000 TIME STEPS

\begin{tabular}{ccc}
\hline & Only 3 sources & sources+deposits \\
\hline Average Planning Time & 1599.8 time steps & 450 time steps \\
\hline Average Satisfaction Level & 59.89 & 88.07 \\
\hline
\end{tabular}

It is important to note that the selection of the resources locations by agents is just an adaptive behavior that begins with an individual decision of an agent during the deposit operation and ends with the maintenance or deletion of this deposit by the entire population. In conclusion, we talk about an individual decisions leading to emerging collective structures. Based on adaptive cognitive map, this experiment allows our CMAS (i) to optimize the planning time of agents and the number of deposits thanks to the learning of the new places of resources, (ii) to improve the adaptive behavior and the level of satisfaction of agents and finally (iii) to leads a fixed number of deposits in fixed places by creation of relevant emergent structures.

\section{CONCLUSION}

In this paper, we brought to light the adaptive capability of the cognitive map, based on bio-inspired model, that enables a multi-robot system to adapt in a unknown environment to solve the navigation task and the deposit problem. We suggested a set of experiments in real robots that show how each robot system is able to learn, adapt and create on-line a cognitive map and how this architecture allows the robots to learn various goals in unknown environments. We also show from experiments on real robots and on simulation that combining learning capabilities with simple rules of taking and deposits and a simple strategy of imitation has a positve feedback atindividual and population levels. As prospects, we are trying to develop the "awareness"of robots/agents in order to allow them to learn the emergent behaviors. In this case, the emergent behavior becomes a "voluntary" behavior. This means that agents could be aware of their own state in the environment. To achieve our goal, we are trying to use an internal observer (IO) [18] based on enactive approach [19]. The IO should allow agents to detect, categorize and create new emergent rules.

\section{ACKNOWLEDGMENT}

The authors would like to thank the financial support of the Tunisian General Direction of Scientific Research and Technological Renovation (DGRSRT), under the ARUB program 01/UR/11 02, the Institut Français de Tunisie, the INTERACT french project referenced ANR_09_CORD_014, the NEUROBOT french project referenced ANR-BLAN-SIMI2-L2-100617-13-01 and DIGITEO project AUTO EVAL.

\section{REFERENCES}

[1] O. Holland and C. Melhuish, "Stigmergy, self-organization, and sorting in collective robotics," Artif. Life, vol. 5, no. 2, pp. 173-202, 1999.

[2] J. L. Deneubourg, S. Goss, N. Franks, A. S. Franks, C. Detrain, and L. Chrétien, "The dynamics of collective sorting robot-like ants and ant-like robots," in Proceedings of the first international conference on simulation of adaptive behavior on From animals to animats. Cambridge, MA, USA: MIT Press, 1990, pp. 356-363.

[3] P. Gaussier and S. Zrehen, "Avoiding the world model trap: An acting robot does not need to be so smart!" Robotics and Computer-Integrated Manufacturing, vol. 11, no. 4, pp. 279 - 286, 1994.

[4] R. Beckers, O. E. Holland, and J.-L. Deneubourg, "From local actions to global tasks: Stigmergy and collective robotics," in In Articial Life IV. Proc. Fourth International Workshop on the Synthesis and Simulation of Living Systems, Cambridge, Massachusetts, USA, 1994, pp. 181-189.

[5] A. Martinoli and F. Mondada, "Collective and cooperative group behaviours: Biologically inspired experiments in robotics," in Experimental Robotics IV, ser. Lecture Notes in Control and Information Sciences, O. Khatib and J. Salisbury, Eds. Springer Berlin / Heidelberg, 1997, vol. 223 , pp. 1-10.

[6] R. U. Muller, M. Stead, and J. Pach, "The hippocampus as a cognitive graph," 1996.

[7] M. E. Hasselmo and H. Eichenbaum, "Hippocampal mechanisms for the context-dependent retrieval of episodes." Neural Netw, vol. 18, no. 9, pp. 1172-1190, Nov. 2005.

[8] L.-E. Martinet, D. Sheynikhovich, K. Benchenane, and A. Arleo, "Spatial Learning and Action Planning in a Prefrontal Cortical Network Model," PLoS Comput Biol, vol. 7, no. 5, May 2011.

[9] J. O'Keefe and L. Nadel, The hippocampus as a cognitive map / John O'Keefe and Lynn Nadel. Clarendon Press ; Oxford University Press, Oxford, 1978.

[10] M. Milford and G. Wyeth, "Mapping a suburb with a single camera using a biologically inspired slam system," IEEE Transactions on Robotics, vol. 24, no. 5, pp. 1038-1053, October 2008.

[11] P. Gaussier, A. Revel, J. P. Banquet, and V. Babeau, "From view cells and place cells to cognitive map learning: processing stages of the hippocampal system.” Biological Cybernetics, vol. 86, no. 1, pp. 15$28,2002$.

[12] A. Alvernhe, T. Van Cauter, E. Save, and B. Poucet, "Different CA1 and CA3 representations of novel routes in a shortcut situation." The Journal of neuroscience : the official journal of the Society for Neuroscience, vol. 28, no. 29, pp. 7324-7333, Jul. 2008.

[13] E. V. Lubenov and A. G. Siapas, "Hippocampal theta oscillations are travelling waves," Nature, vol. 459, no. 7246, pp. 534-539, May 2009.

[14] D. M. Smith and S. J. Y. Mizumori, "Hippocampal place cells, context, and episodic memory," Hippocampus, pp. 716-729, 2006.

[15] J. P. Banquet, P. Gaussier, J. C. Dreher, C. Joulain, A. Revel, and W. Gunther, "Spacetime, order and hierarchy in fronto-hippocamal system : A neural basis of personality. in cognitive science perspectives on personality and emotion." Elsevier Science BV, p. 123189, 1997.

[16] M. Dorigo, E. Bonabeau, and G. Theraulaz, "Ant algorithms and stigmergy," Future Gener. Comput. Syst., vol. 16, no. 9, pp. 851-871, June 2000.

[17] P. Laroque, N. Gaussier, N. Cuperlier, M. Quoy, and P. Gaussier, "Cognitive map plasticity and imitation strategies to improve individual and social behaviors of autonomous agents," Journal of Behavioral Robotics, 2010.

[18] J. Tani, "An interpretation of the "self" from the dynamical systems perspective: A constructivist approach," Journal of Consciousness Studies, vol. 5, pp. 516-542, 1998.

[19] F. Varela, E. Thompson, E. Rosch, and A. Rangarajan, "Book review: The embodied mind: Cognitive science and human experience by $\mathrm{f}$. varela, e. thompson and e. rosch," 1991. 